\title{
Análisis de emisiones contaminantes y consumo de combustible a diferentes proporciones y marcas de aceites lubricantes para motores de dos tiempos empleando gasolina extra y super
}

DOI: https://doi.org/10.33262/ap.v3i3.1.93

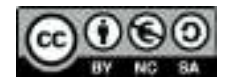

Fuel consumption and pollutant emissions analysis at different proportions and lubricating oils brands for two-stroke engines using extra and super gasoline

Victor David Bravo Morocho. ${ }^{1}$, Edison Patricio Abarca Pérez. ${ }^{2}$ \& Andrés Daniel Cárdenas Valencia. ${ }^{3}$

\begin{abstract}
.
The consumption of extra and super fuels in combination with three brands of oils A, B and $\mathrm{C}$, and the exhaust gas emissions in a 171 cc two-stroke engine were analyzed at different proportions. First, three brands of coded oils were defined as, A (Motorex), B (Golden Bear) and C (Motul), and the extra and super fuels. Each brand of oil was mixed with the two types of gasoline, applying a mixture formula with the help of the oil manufacturer's manual and laboratory equipment to obtain exact quantities, thus obtaining six different mixtures or combinations. For fuel consumption tests, 5 samples were taken from each combination and the two-stroke engine was run at idle for 20 minutes and the consumption of each combination was determined, resulting in the $\mathrm{C}$ oil

1 Escuela Superior Politécnica de Chimborazo, Facultad de Mecánica. Riobamba, Ecuador. victor.bravo@espoch.edu.ec

2 Escuela Superior Politécnica de Chimborazo, Facultad de Mecánica. Riobamba, Ecuador. edison.bravo@espoch.edu.ec

3 Escuela Superior Politécnica de Chimborazo, Facultad de Mecánica. Riobamba, Ecuador. andres.cardenas@espoch.edu.ec
\end{abstract}


(Motul) whose 25: 1 ratio, generates lower fuel consumption. For the exhaust gas analysis, the free acceleration method of the INEN 2202 standard was applied, which allowed observing the coloration and opacity of the exhaust gases of each mixture, resulting in C Oil (Motul) reducing fuel consumption. and it has an average opacity of $60 \%$ if it is mixed with the extra gasoline, in turn, said opacity is reduced to $40 \%$ when the mixture is with the super gasoline. The consumption results were contrasted with the ANOVA statistical test in MINITAB obtaining a p value $(1.617 \mathrm{E}-20)<\alpha(0.05)$ accepting the alternative hypothesis, supported by a qualitative analysis for the gas results

Keywords: Two-stroke engine, combination of fuel and oil, fuel consumption, exhaust gases, opacity, two-stroke engine oil.

\section{Resumen.}

Se analizo el consumo de combustibles extra y super en combinación con tres marcas de aceites A, B y C, y las emisiones de gases de escape en un motor de dos tiempos de 171 cc a diferentes proporciones. Primero se definió tres marcas de aceites codificadas como, A (Motorex), B (Golden Bear) y C (Motul), y los combustibles extra y súper. Cada marca de aceite fue mezclada con los dos tipos de gasolina, aplicando una fórmula de mezclas con ayuda del manual del fabricante del aceite y equipos de laboratorio para obtener cantidades exactas, obteniendo así seis mezclas o combinaciones diferentes. Para las pruebas de consumo de combustible, se tomaron 5 muestras de cada combinación y se puso en funcionamiento el motor de dos tiempos en ralentí durante 20 minutos y se determinó el consumo de cada combinación, dando como resultado que el aceite $\mathrm{C}$ (Motul) cuya proporción de 25:1, genera menor consumo de combustible. Para el análisis de gases de escape, se aplicó el método de aceleración libre de la norma INEN 2202, el cual permitió observar la coloración y opacidad de los gases de escape de cada mezcla resultando que el Aceite $\mathrm{C}$ (Motul) reduce el consumo de combustible y presenta una opacidad media de $60 \%$ si se mezcla con la gasolina extra a su vez dicha opacidad se reduce a un $40 \%$ cuando la mezcla es con la gasolina súper. Los resultados de consumo fueron contrastados con la prueba estadística de ANOVA en MINITAB obteniendo un $\mathrm{p}$ valor $(1.617 \mathrm{E}-20)<\alpha(0.05)$ aceptando la hipótesis alternativa, apoyado de un análisis cualitativo para los resultados de gases.

Palabras claves: Motor de dos tiempos, combinación de combustible y aceite, consumo de combustible, gases de escape, opacidad, aceite para motor de dos tiempos.

\section{Introducción.}

Este trabajo experimental se centra en establecer diferencias en el consumo de combustible y el análisis del comportamiento de las emisiones contaminantes en un motor de dos tiempos, para ello se establece tres marcas de aceites para motores de dos tiempos existentes en el mercado. Existen estudios donde solo con las variables empleadas se 
reduce el humo en motores dos tiempos (Cárdenas Valencia, 2021). En Holanda se mantuvo la proporción de aceite al $1 \%$ usando aceite vegetal oxigenado con el cual se redujo el humo entre un 50 y $70 \%$ en un motor de dos tiempos. (Singh,2011).

Cada marca de aceite con su proporción de mezcla definida será puesta a prueba tanto para la gasolina extra como súper, de esta manera se aplicará la mezcla en el motor y en un tiempo constante definido para todas pruebas, se tomará la cantidad inicial y final de la mezcla as así se obtendrá la mezcla consumida por el motor. En cuanto a las emisiones con un análisis de campo se determinará visualmente el comportamiento de estas en el motor aplicando las definiciones y el método de aceleración libre de la Norma Técnica Ecuatoriana (INEN, 2013), al emplear una técnica visual se puede observar la coloración del humo en motores dos tiempos (Cárdenas Valencia, 2021). Según (Pesis,2015) una coloración azulada será alta presencia de aceite en el proceso de combustión y una coloración blanco-azulado poca presencia de aceite en el proceso.

Para determinar la variación que se produzca o no en la hipótesis de acuerdo a las variables empleadas se aplicará la prueba estadística de ANOVA del software minitab, el cual compara si en los diferentes grupos de las pruebas de consumo de combustible existe o una diferencia significa (Minitab, 2020), además esta validación será apoyada por una análisis de campo donde vidualmente por medio de figuras y graficas se determinara una coloración y opacidad visual de los gases de escape de las diferentes mezclas de combustible empleadas. Ambos resultados serán cotejados para determinar conclusiones sobre la incidencia o no de la calidad en los productos empleados (Cárdenas Valencia, 2021).

\section{Modo de lubricación en un motor de dos tiempos.}

En la mayoría de los motores especialmente en motocicletas el aceite se añade al combustible, manipulando la mezcla de manera externa en el tanque de combustible, cuando la mezcla ingresa al interior se pega en las paredes y en todas las partes móviles donde se realiza la lubricación. Este es un tipo de lubricación que se denomina lubricación por mezcla o engrase directo. Se considera que la cantidad correcta de aceite a introducir en el combustible está entre un $2 \%$ a $5 \%$. El operador debe estar capacitado para realizar la proporción correcta de la mezcla. (Secundino, 2011: p.279).

Es decir que al no disponer de un sistema de lubricación como se lo tiene en un motor de cuatro tiempos, el aceite ingresará por la lumbrera de admisión junto con la mezcla y desde ahí iniciará la lubricación al motor a sus elementos, este proceso se renovará constantemente cada vez con el fin de mantener lubricado al motor, en la expulsión de los gases también está presente el aceite, es por ello la cantidad de humo muchas veces de color azul que presentan los motores de dos tiempos. En la figura 1, se ilustra el proceso continuo de lubricación. (Cárdenas Valencia, 2021) 
Figura 1

Proceso de lubricación en un motor de dos tiempos

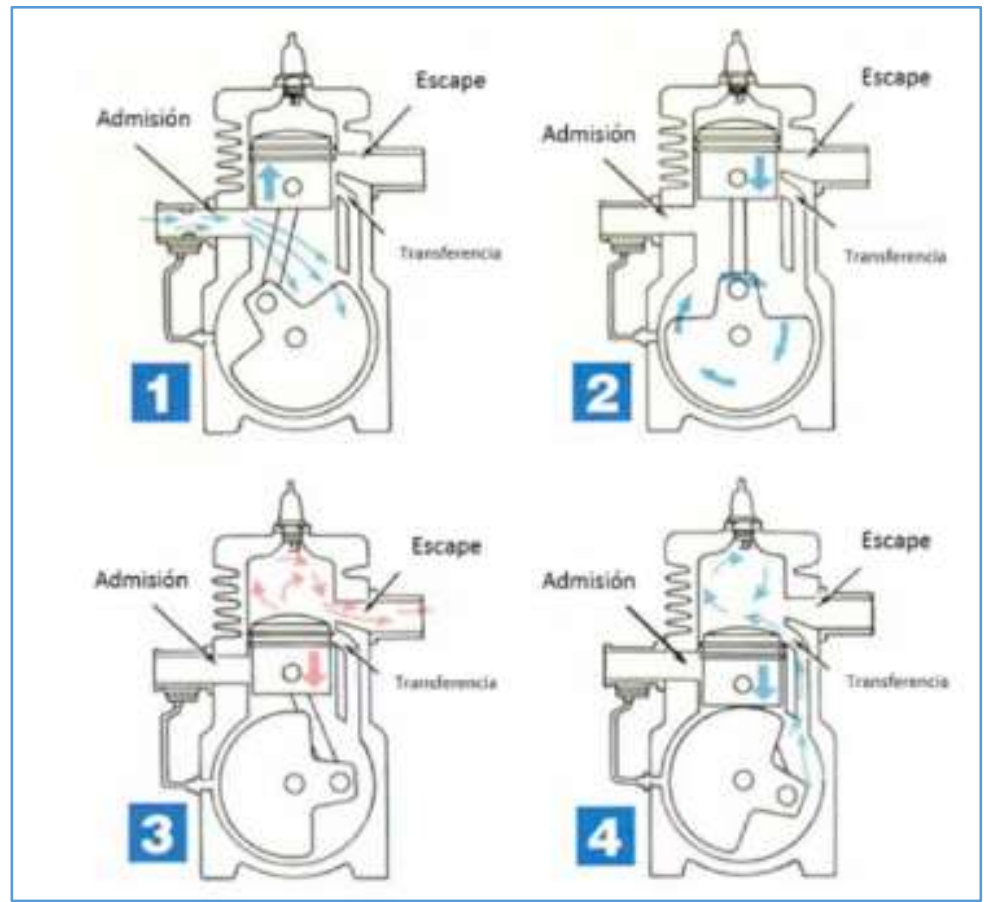

Fuente: Bardahl. 2015.

\section{Análisis de emisiones de escape por coloración de humo.}

La interpretación del color de humo de escape puede indicar el comportamiento del motor, la manera y los agentes que se involucran en el proceso de combustión, a continuación, se presentan algunos casos, (Cárdenas Valencia, 2021):

- Ligero de color blanco: En motores dos tiempos su presencia es normal debido a la condensación del aire caliente, no se trata necesariamente de agua en el proceso de combustión. (Pesis,2015. p.139).

- Denso de color blanco: En motores dos tiempos con sistemas de refrigeración por líquido, se debe por la presencia de líquido refrigerante o agua en el cilindro debido a problemas en la junta o en el bloque. (Pesis,2015. p.139).

- Denso blanco-azulado: Se debe a una ligera presencia de aceite en el proceso de combustión, generalmente por falta de estanqueidad en guías de válvulas o en los segmentos del pistón, o procesos normales de funcionamiento en motores dos tiempos. (Pesis,2015. p.139).

- Azulado: En motores de dos tiempos, es muy normal esta coloración, la intensidad de humo azul se verá reflejada por la cantidad de aceite quemado en el proceso de combustión. (Cárdenas Valencia, 2021)

- Denso de color oscuro: Se debe al exceso de combustible en el proceso de combustión, es decir una mezcla excesivamente rica, se puede presentar por un problema en el sistema de alimentación. (Pesis,2015. p.139). 


\section{Norma INEN 2202.}

Es una norma ecuatoriana para la gestión ambiental en automotores la cual permite determinar la opacidad de una manera visual en los motores que no se puede determinar sus emisiones contaminantes por medio de la utilización de equipos, es el caso de los motores dos tiempos, por el riesgo de dañar equipos de diagnósticos por el aceite que emanan (Cárdenas Valencia, 2021).

Esta norma aplica el método de aceleración libre que consiste en aumentar las revoluciones del motor rápidamente desde mínimas a máximas revoluciones y observar el comportamiento de los gases visualmente (INEN 2202,2013).

\section{Metodologia.}

Se implementa una investigación de campo donde se recolectan y se registran ordenadamente datos nuevos productos del objeto de estudio, ayudándose de la técnica de la observación. (Baena,2014. p.12). El objetivo es obtener resultados de consumo de combustible, opacidad y coloración de gases de escape de un motor dos tiempos, para ello se han empleado tres marcas de aceites para motores dos tiempos codificadas como A (Motorex), B (Golden Bear) y C (Motul), que han sido mezcladas con dos calidades de combustibles definidas por su octanaje extra (87 octanos) y súper (92 octanos), de esta manera se obtuvieron seis distintas mezclas de combustibles que serán analizadas en un banco de pruebas de un motor de dos tiempos. Para las pruebas de consumo y gases se estableció un protocolo de pruebas. Dentro de cada prueba se han realizado cinco muestras. Es importante recalcar que las pruebas experimentales ejecutadas se realizaron en el país Ecuador, Ciudad de Ambato a una presión atmosférica de (72 Kpa), y a una altitud de (2500) msnm. (Cárdenas Valencia, 2021)

\section{Equipos.}

Para determinar las propiedades mecánicas del cabezote de referencia se realiza las 4 pruebas fundamentales para caracterizar el material, siendo el ensayo de metalografía, micrografía, espectrometría y dureza como se muestra a continuación (Cárdenas Valencia, 2021).

Se han utilizado materiales normados como: jarras de precipitación, jeringas, medidores de nivel para la superficie de las jarras con el fin de minimizar los errores de medición, se destaca el uso del banco de pruebas de un motor de dos tiempos, sus características se presentan en la tabla 1. (Cárdenas Valencia, 2021) 
Figura 2

Banco de pruebas motor dos tiempos.

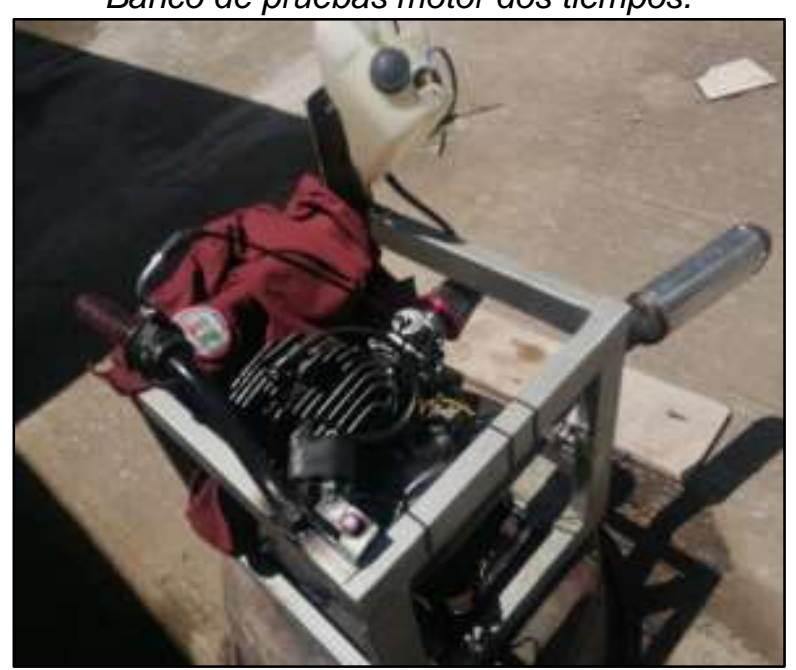

Fuente: Autores. 2021.

A continuación, en la Tabla 1, se muestra los datos técnicos del motor de pruebas.

Tabla 1

Especificaciones técnicas motor de dos tiempos de pruebas.

\begin{tabular}{cc}
\hline \multicolumn{2}{c}{ DATOS TECNISO DEL MOTOR } \\
\hline Cilindrada & $171 \mathrm{cc}$ \\
Número de Cilindros & Monocilindrico 2 tiempos \\
Diámetro x carrera & $66 \times 50 \mathrm{~mm}$ \\
Relación de compresión & $6,7: 1$ \\
Potencia & $16.00 \mathrm{cv}(11.7 \mathrm{kw})$ a $7000 \mathrm{rpm}$ \\
Refrigeración & Aire \\
Arranque & Pedal \\
\hline
\end{tabular}

Fuente: Autores. 2021.

Fórmula para la formulación de las mezclas combustible -aceite.

Para formar las seis distintas mezclas, con los aceites codificados como A, B, C se han aplicado las proporciones recomendadas por sus fabricantes las cuales fueron 40:1 ,50;1,25:1 respectivamente tanto para la gasolina extra como súper (Cárdenas Valencia, 2021). La fórmula aplicada fue la siguiente:

Para calcular correctamente las cantidades se ha definido la siguiente formula:

Cantidad de aceite $=\frac{\text { cantidad de combustible a preparar } * 1}{\text { proporción definida de combustible }}$

(1)

La cantidad obtenida de aceite a partir de la fue añadida a una cantidad constante de gasolina extra y súper obteniendo un total de 6 mezclas diferentes. En la siguiente tabla se resume los cálculos obtenidos, (Cárdenas Valencia, 2021): 
Tabla 2

Resumen de proporciones obtenidas mediate cálculo. COMBINACIONES DE COMBUSTIBLE Y ACEITE

\begin{tabular}{cccc}
\hline Tipo de Mezcla & Proporciones & Cantidad combustible $(\mathrm{ml})$ & $\begin{array}{c}\text { Cantidad } \\
\text { aceite }(\mathrm{ml})\end{array}$ \\
\hline 1.Extra-Aceite A & $40: 1$ & 2000 & 50 \\
2.Extra-Aceite B & $50: 1$ & 2000 & 40 \\
3.Extra-Aceite C & $25: 1$ & 2000 & 80 \\
4.Super-Aceite A & $40: 1$ & 2000 & 50 \\
5.Super-Aceite B & $50: 1$ & 2000 & 40 \\
6.Super-Aceite C & $25: 1$ & 2000 & 80 \\
\hline
\end{tabular}

Fuente: Autores. 2021.

\section{Proceso de formación de las mezclas.}

Aplicando los cálculos obtenidos en la tabla 2, con la ayuda de un embudo y la jarra graduada colocar en un envase 2 litros de combustible. Tomar con una jeringa la cantidad correspondiente de aceite, si no se puede directamente ayudarse de otro envase. Introducir con la jeringa el aceite en el envase de combustible. Agitar el envase para que se mezclen los componentes y notar la diferencia en la coloración después de realizar la mezcla. Los procesos se repiten para las diferentes mezclas. Finalmente se obtuvo 6 tipos diferentes de mezclas, con diferentes proporciones ,3 para gasolina extra y 3 para gasolina súper como se observa en la Figura 3, a continuación. (Cárdenas Valencia, 2021)

Figura 3

Mezclas preparadas para la prueba experimental.

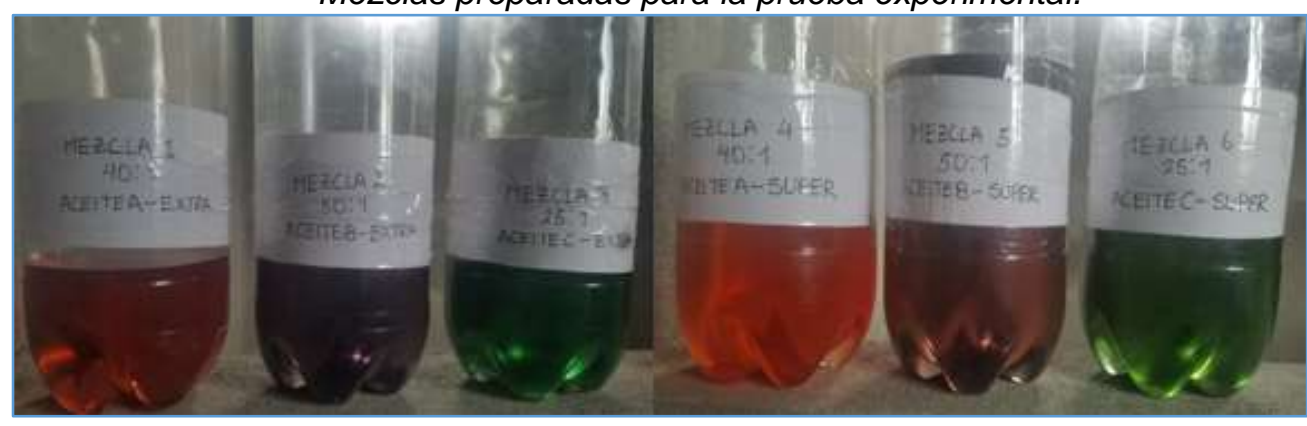

Fuente: Autores 2021.

\section{Protocolo para la ejecución de las pruebas de consumo}

Las seis mezclas obtenidas de combustible al aplicar el cálculo correspondiente son puestas a prueba en el motor de dos tiempos. El tiempo de cada prueba para cada mezcla son veinte minutos. Se colocará una cantidad de volumen inicial en el tanque de combustible y se medirá el volumen final, así se obtendrá el volumen consumido de cada 
mezcla. Se tomarán cinco muestras en cada prueba. Se deben aplicar los reglajes previos del motor, como chequeo de bujía, regulación de ralentí estable, y temperatura optima de funcionamiento. Para ejecutar las pruebas de consumo se aplica el siguiente protocolo: (1) Si existe una mezcla previa diferente a la que se va a realizar en la prueba, vaciarla por el tornillo de la cuba del carburador y colocar la mezcla correspondiente a la prueba en el tanque de combustible, (2) Colocar $500 \mathrm{ml}$ referencial de la mezcla graduados en una jarra, en el tanque de combustible, (3) Encender el motor y comenzar la prueba cronometrada a 20 minutos. Durante la prueba, observar que el comportamiento del motor sea estable, caso contrario si sufre algún desperfecto, fuga de combustible o se apaga la prueba quedará invalidada. Aproximadamente a los 10 minutos de prueba corrida, encender el ventilador para que la corriente de aire circule por las aletas del motor. Al finalizar los 20 minutos de prueba, apagar el motor, apagar el ventilador, retirar la manguera que conecta el tanque de combustible al carburador y vaciar la mezcla en la jarra graduada. Visualizar y registrar la cantidad obtenida en cada muestra, aplicar los mismos pasos para todas las 6 diferentes pruebas (Cárdenas Valencia, 2021).

\section{Protocolo para la ejecución de las pruebas de gases}

En este caso se aplican los mismos reglajes previos de las pruebas de consumo. Para está prueba se aplica los conceptos y definiciones de la prueba de aceleración libre de la norma técnica ecuatoriana 2202 para opacidad, el fin es observar visualmente la coloración y la opacidad de los gases de un motor de dos tiempos, al ser un método de campo se fijan los siguientes parámetros y pasos : (1) La coloración será observada entre : Denso color blanco, Blanco-azulado ,Azulado,Oscuro, (2) Los parámetros de opacidad serán : Muy alta(100\%), Alta(80\%),Media(60\%),Baja(40\%),Muy baja(20\%),(3) En cada prueba se toman 5 mediciones, de acuerdo a los intervalos y aplicación del método de aceleración libre. Según la norma (INEN 2202,2013) el método de aceleración libre es aumentar las revoluciones del motor rápidamente desde mínimas a máximas revoluciones, (4) Corroborar que no existan obstáculos en el accionamiento del acelerador para que la prueba sea válida. (INEN 2202,2013), (5) Encender el motor y aplicar el método de aceleración libre este consiste en mantener oprimido el acelerador durante 2 segundos, después soltar el acelerador para que el motor regresar al estado de ralentí. (INEN 2202,2013), (6) El motor debe ser sometido por lo menos a cuatros ciclos durante el ensayo de aceleración libre, el tiempo de espera para cada intervalo debe ser de 10 a 12 segundos con el fin de que durante este tiempo el motor se vuelva a estabilizar. (INEN 2202,2013), (7) Durante la ejecución de la prueba, registrar el comportamiento de los gases de escape, su coloración y la intensidad de la opacidad de acuerdo con los parámetros establecidos, (8) Finalmente, para iniciar una prueba con una mezcla diferente se debe repetir nuevamente todo el protocolo desde su inicio, con el objetivo de aplicar los mismos pasos para todas las 6 diferentes pruebas (Cárdenas Valencia, 2021).

\section{Resultados.}

Una vez aplicada la metodología se procesan los datos para ser interpretados. En la tabla 3 se presentan los resultados de las pruebas de consumo que son analizados 
cualitativamente mediante la prueba estadística de ANOVA en minitab. (Cárdenas Valencia, 2021)

Tabla 3

Datos obtenidos en las pruebas de consumo en intervalo de tiempo constante. Pruebas de consumo realizas con las seis diferentes mezclas Combustible-Aceite

\begin{tabular}{lrrrrrr}
\hline $\begin{array}{l}\text { Mez } \\
\text { clas }\end{array}$ & $\begin{array}{l}\text { 1.Extra/Ac } \\
\text { eite A }(\mathrm{ml})\end{array}$ & $\begin{array}{l}\text { 2.Extra/Ace } \\
\text { ite B }(\mathrm{ml})\end{array}$ & $\begin{array}{l}\text { 3.Extra/Acei } \\
\text { te } \mathrm{C}(\mathrm{ml})\end{array}$ & $\begin{array}{l}\text { 4.Super/Ace } \\
\text { ite A }(\mathrm{ml})\end{array}$ & $\begin{array}{l}\text { 5.Super/Ace } \\
\text { ite B }(\mathrm{ml})\end{array}$ & $\begin{array}{l}\text { 6.Super/Ace } \\
\text { ite } \mathrm{C}(\mathrm{ml})\end{array}$ \\
\hline 1 & 330 & 360 & 220 & 330 & 290 & 275 \\
2 & 320 & 345 & 225 & 340 & 310 & 285 \\
3 & 325 & 360 & 215 & 340 & 290 & 280 \\
4 & 340 & 355 & 215 & 330 & 305 & 280 \\
5 & 320 & 350 & 220 & 335 & 295 & 280 \\
\hline
\end{tabular}

Fuente: Autores. 2021.

Una vez validado los datos se procede a realizar el análisis estadístico de ANOVA asumiendo distribución normal y varianzas iguales, el nivel de significancia es $\alpha=0.05$ mientras que el valor $\mathrm{p}$ value de minitab $=1.617 \mathrm{E}-20$, este valor significa que hay un alto nivel de significancia para la selección de la hipótesis que existe una diferencia en los grupos , como se observa en la figura 1 el traslape que existe entre las diferentes mezclas puestas a prueba en la investigación y se puede evidenciar que la mezcla de combustible Extra-Aceite $\mathrm{C}$ es quien tiene una diferencia significativa en cuanto a las demás ya que es la que presenta el menor valor en cuanto a la media de consumo de combustible. (Cárdenas Valencia, 2021)

Gráfico 1

Análisis de consumo de combustible.

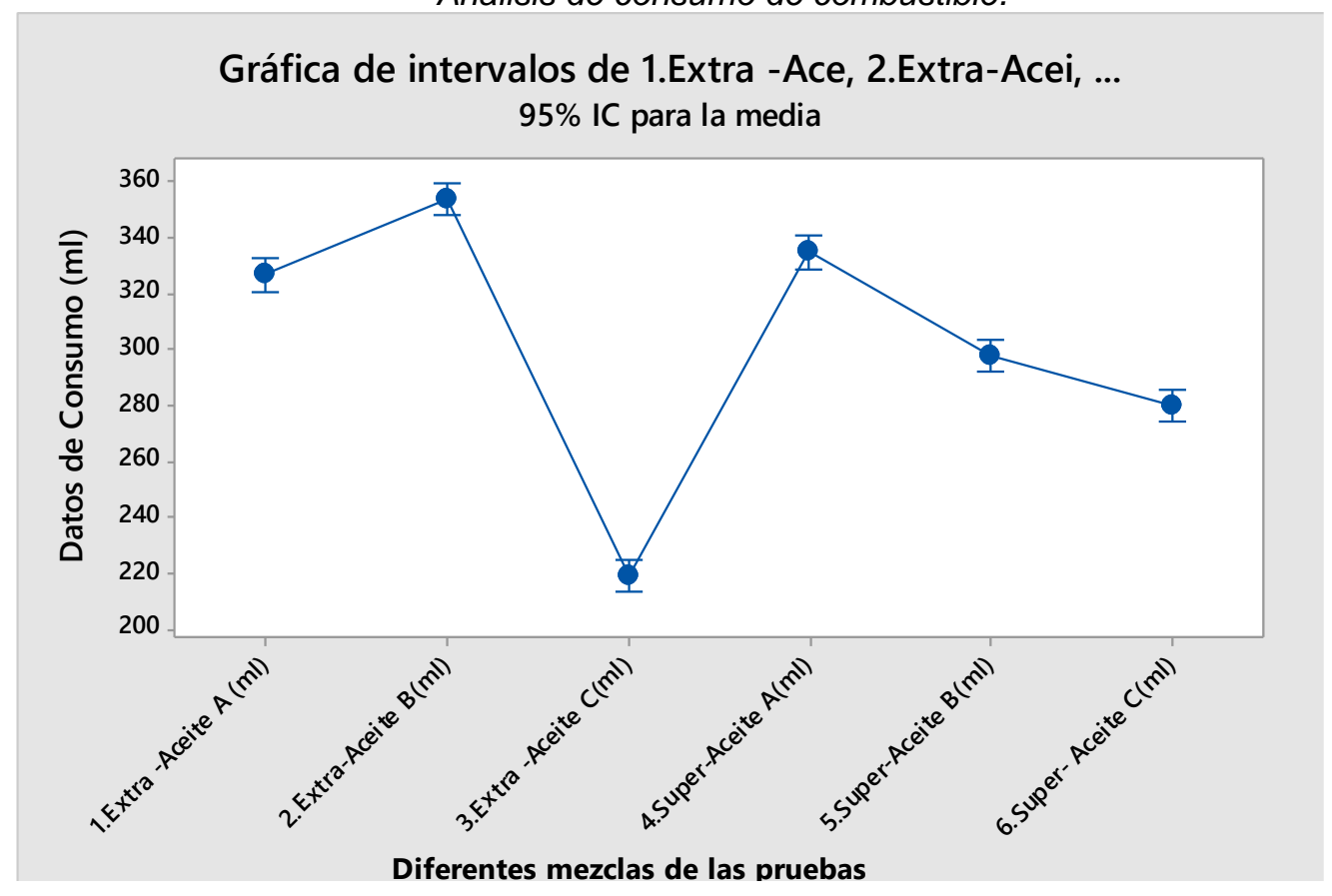

Fuente: Autores 2021 
Para confirmar y validar dichos resultados se cotejó los resultados aplicando un protocolo para las pruebas de emisiones contaminantes, en las siguientes figuras a continuación se observa los resultados (Cárdenas Valencia, 2021):

Figura 4

Coloración y opacidad prueba: Extra y Aceite A

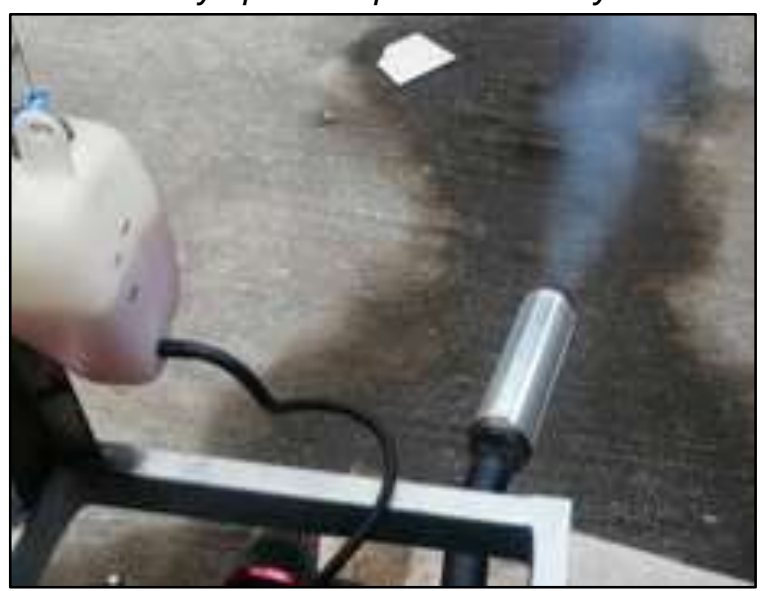

Fuente: Autores 2021

Figura 5

Coloración y opacidad prueba: Extra y aceite $B$

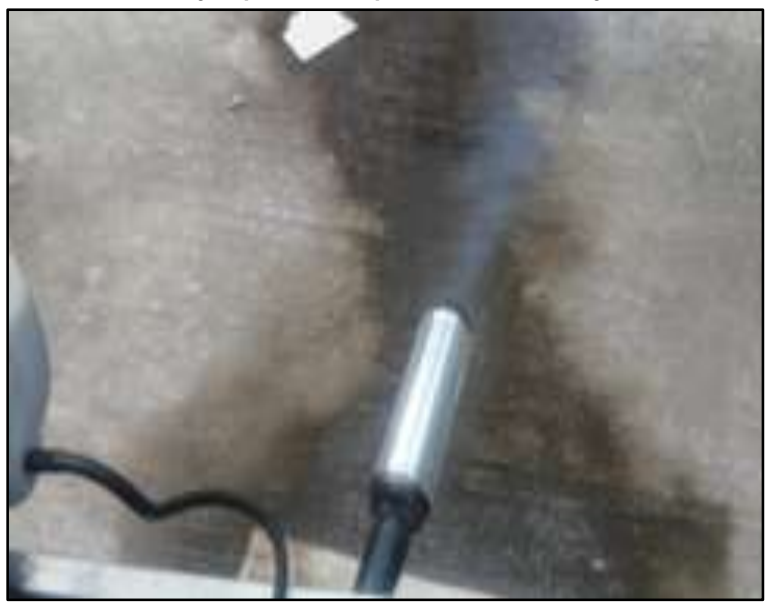

Fuente: Autores 2021.

Figura 6

Coloración y opacidad prueba: Extra y aceite $C$

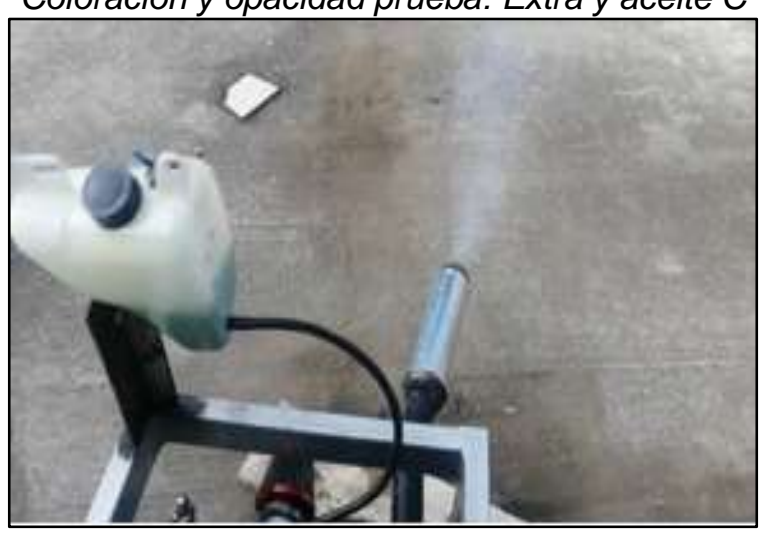

Fuente: Autores 2021. 
Figura 7

Coloración y opacidad prueba: Super y aceite $A$

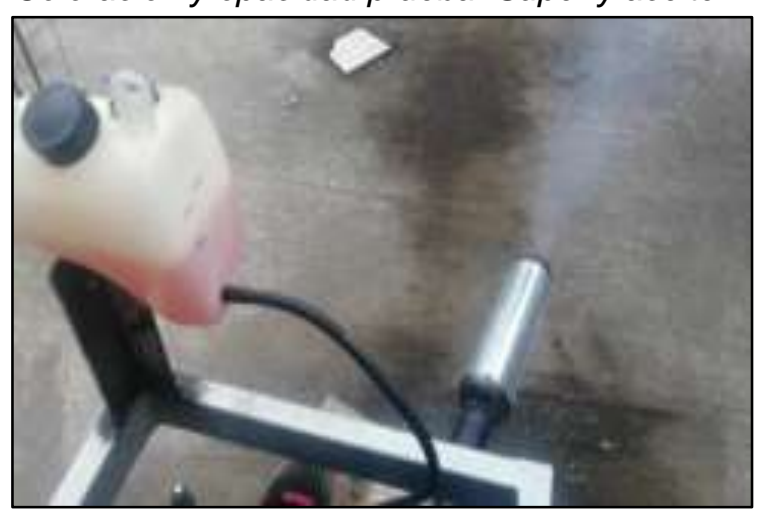

Fuente: Autores 2021.

\section{Figura 8}

Coloración y opacidad prueba: Super y aceite $B$

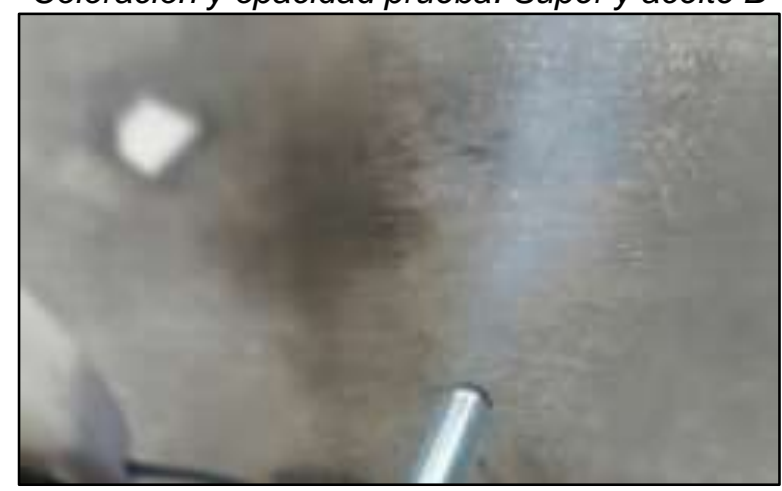

Fuente: Autores 2021.

\section{Figura 9}

Coloración y opacidad prueba: Super y aceite $C$

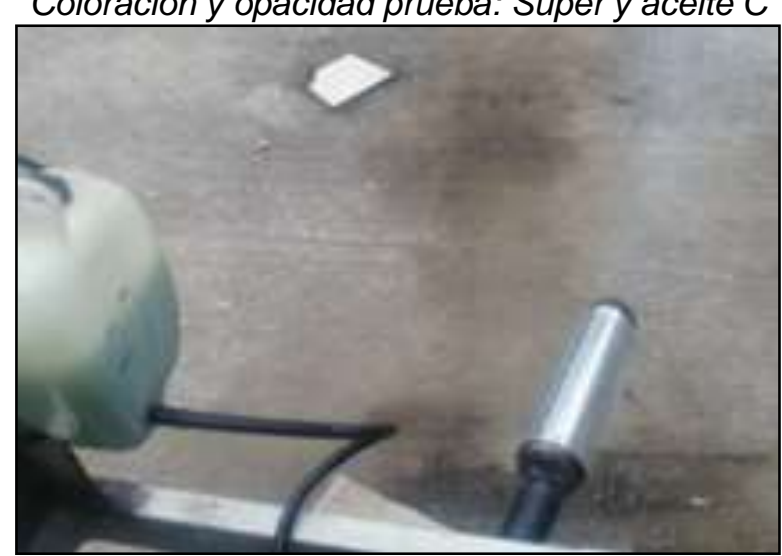

Fuente: Autores 2021

En el gráfico 2 a continuación, se puede observar un análisis cualitativo para cotejar los resultados. (Cárdenas Valencia, 2021) 
Gráfico 2

Análisis coloración-opacidad de las emisiones de un motor $2 T$

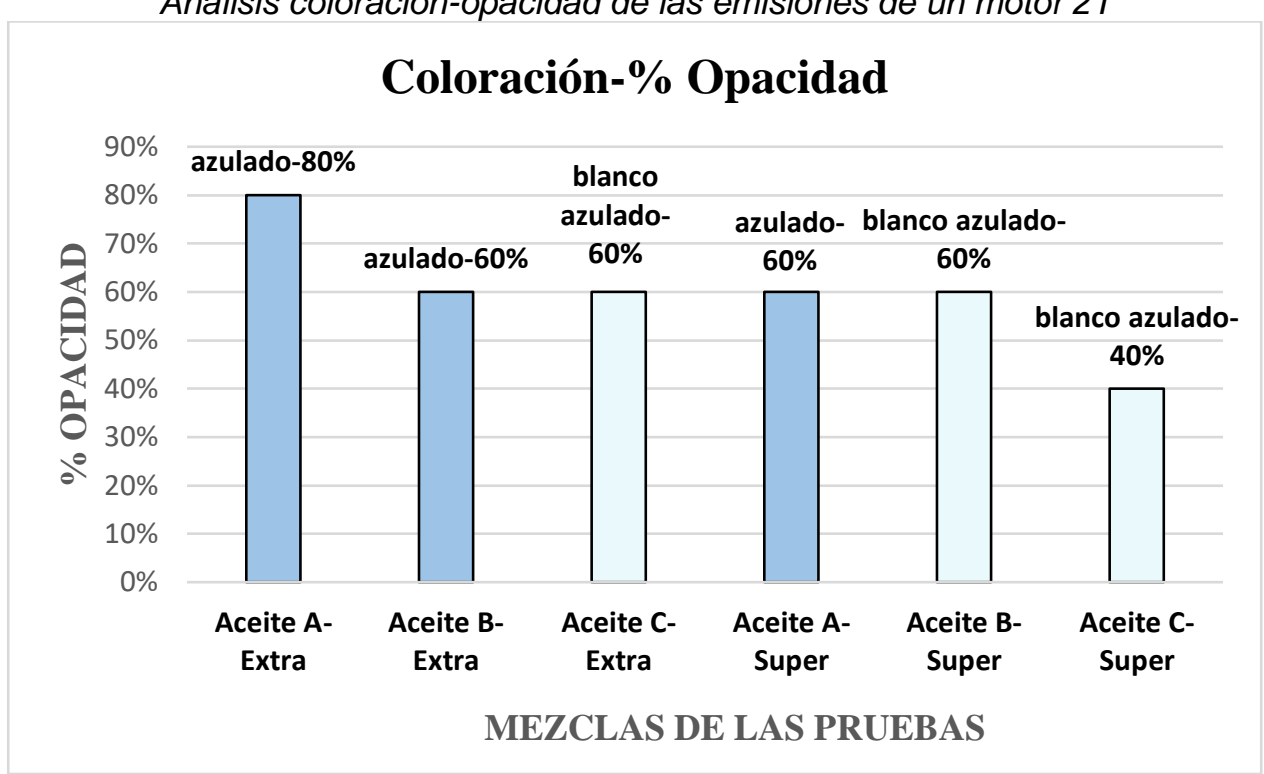

Fuente: Autores 2021

Se puede observar que mezcla Extra -Aceite A, presenta una mayor opacidad en relación con el resto con un color azulado lo que significa que hay una presencia considerable de cantidad de aceite en el proceso de combustión en la salida de los gases de escape. (Cárdenas Valencia, 2021)

La mezcla quien presenta una menor opacidad es la Súper-Aceite A con un porcentaje de $40 \%$ a su vez una coloración blanco-azulado se interpreta como una presencia leve de aceite en el proceso de combustión, otras mezclas con una presencia leve de aceite en la combustión son las mezclas Extra-Aceite C y Súper-Aceite B ambas con un color blanco azulado y una opacidad de 60\%. (Cárdenas Valencia, 2021)

Una diferencia significativa que se puede notar con el análisis de coloración y opacidad en los gases es que el uso de la gasolina súper genera un cambio en el comportamiento de los gases de escape, que fueron los siguientes, (Cárdenas Valencia, 2021):

- El Aceite A (Motorex) cambia de una opacidad alta de $80 \%$ con la gasolina extra a una opacidad media de $60 \%$ con la gasolina súper manteniendo el color azulado de presencia de aceite considerable en la combustión. (Cárdenas Valencia, 2021)

- El Aceite B(Golden Bear) cambia de un color azulado con gasolina extra a un color blanco-azulado con la gasolina súper lo que significa que en la combustión existe menor presencia de aceite. (Cárdenas Valencia, 2021)

- El Aceite C (Motul) mantiene el color blanco-azulado con las gasolinas extra y súper sin embargo con la gasolina súper se reduce la opacidad de $60 \%$ a $40 \%$, lo que indica el buen comportamiento y aplicación de este aceite en las mezclas de combustible. (Cárdenas Valencia, 2021) 


\section{Conclusiones.}

- El Aceite C (Motul) cumple con especificación y características de calidad descritas por el fabricante, en efecto reduce el consumo de combustible en un $40 \%$ en promedio respecto de los aceites Ay B y presenta una opacidad media de $60 \%$ si se mezcla con gasolina extra a su vez dicha opacidad se reduce a un $40 \%$ cuando la mezcla es con gasolina súper.

- Del análisis de las emisiones de escape de un motor dos tiempos se ha evidenciado como al emplear la gasolina súper en las mezclas de las distintas pruebas, existe una variación en la coloración y emisión de las emisiones de escape del motor siendo el aceite $\mathrm{C}$ (Motul) un aditivo ideal para reducir la opacidad y el consumo de combustible con respecto a los aceites A y B.

- Una vez analizado el consumo de combustible por medio del estadístico ANOVA y el comportamiento de los gases de escape por medio de gráficas, los resultados se complementan y se corrobora la hipótesis alterna planteada en la investigación con un p valor $(1.617 \mathrm{E}-20)<\alpha(0.05)$, dando una óptima proporción en la mezcla aceite -combustible siendo de 25:1 para el aceite $C$, mejorando los resultados de eficiencia de consumo de combustible y reduce la emanación de los gases de escape.

\section{Referencias bibliográficas.}

Baena, P. Metodología de la Investigación [en línea] .México: Grupo Editorial Patria.,2014. Disponible en: https://elibro.net/es/ereader/espoch/40362

Cárdenas Valencia,Andrés Daniel. Análisi de emisiones contamiantes y consumo de combustible a diferentes proporciones y marcas de aceites lubriacantes para motores de dos tiempos empleando gasolina extra y super. Escuela Superior Politécnica de Chimborazo ESPOCH.2021

Minitab. Métodos y fórmulas para el análisis de varianza en ANOVA de un solo factor. En línea] 2020. Disponible en: https://support.minitab.com/es$\mathrm{mx} / \mathrm{minitab} / 18 /$ help-and-how-to/modeling-statistics/anova/how-to/one-wayanova/methods-and-formulas/analysis-of-variance/\#mean-squares-ms

NTE INEN 2202. Gestión Ambiental aire vehiculos automotores determinación de la opacidad de emisiones de escape de motores diesel mediante la prueba metodo de

aceleracionlibre.Disponibleen:https://www.normalizacion.gob.ec/buzon/normas 12202-1.pdf.

Pesis, H. Motos: motores de 2 y 4 tiempos [en línea]. $1^{\mathrm{a}}$ ed. Buenos Aires: Fox Andina,S.A.,2015.Disponible en: https://books.google.com.ec/books?id=bTimDAAAQBAJ\&pg=PA139\&dq=col or+de+humo+de+escape \&hl=es419\&sa=X\&ved=2ahUKEwiIvODY_6buAhUx11kKHc- 
EDKoQ6wEwAHoECAQQAQ\#v=onepage \&q=color\%20de\%20humo\%20de\% 20escape $\& \mathrm{f}=$ false

Secundino, E. Motores [en línea]. Madrid: Macmillan Iberia,S.A.,2011.Disponible en: https://elibro.net/es/ereader/espoch/101844

Singh, A. K. "Castor oil-based lubricant reduces smoke emission in two-stroke engines". Industrial crops and products [en línea] ,2011, (Holanda), vol. 33, no 2, p. 287295.Disponible en https://www.sciencedirect.com/science/article/abs/pii/S0926669010003341

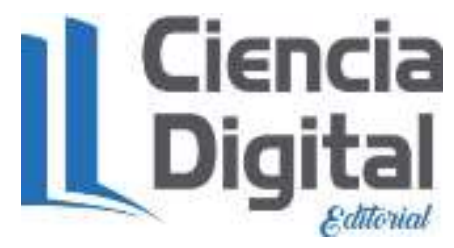




\section{PARA CITAR EL ARTÍCULO INDEXADO.}

Bravo Morocho, V. D., Abarca Pérez, E. P., \& Cárdenas Valencia, A. D. (2021). Análisis de emisiones contaminantes y consumo de combustible a diferentes proporciones y marcas de aceites lubricantes para motores de dos tiempos empleando gasolina extra y super. AlfaPublicaciones, 3(3.1), 289-303. https://doi.org/10.33262/ap.v3i3.1.93

\section{LCiencia}

El artículo que se publica es de exclusiva responsabilidad de los autores y no necesariamente reflejan el pensamiento de la Revista Conciencia Digital.

El artículo queda en propiedad de la revista y, por tanto, su publicación parcial y/o total en otro medio tiene que ser autorizado por el director de la Revista Conciencia Digital.

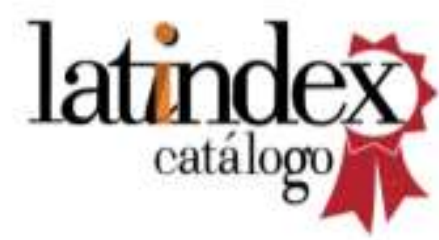

